\title{
Decoupling Control Analysis of a Flux Reversal Linear Rotary Permanent Magnet Actuator
}

\author{
Kaikai Guo †, Youguang Guo, Jingzhao Li
}

\begin{abstract}
In order to meet the requirement of industrial production, a control system of a novel flux reversal linear rotary permanent magnet actuator (FR-LRPMA) is developed in this paper, and the electromagnetic and motion decoupled process is analyzed in detail. Firstly, the control equation of FR-LRPMA is derived in the nine phase stator coordinate system and dual $d q$-axis mover coordinate system. Then the control model of FR-LRPMA is built in MATLAB/Simulink using improved space vector pulse width modulation (SVPWM), and the linear, rotary and spiral motions of the proposed actuator are achieved. Finally, the prototype of the actuator is manufactured, and the experiment platform of FR-LRPMA is set up to measure the cogging torque and linear detent force, validating the simulation results. It is concluded that the control system of FR-LRPMA can be converted into two independent single freedom motors by dual $d q$ transformation. The improved SVPWM is effective in the control system, and the method can be used to solve the electromagnetic and motion decoupling problems.
\end{abstract}

Keywords: Decoupling control, dual $d q$ transformation, flux reversal, linear rotary actuator

\section{Introduction}

The study of two-degree-of-freedom linear rotary motor (2-DOF LRM) has been an important research topic, especially in the recent years. Many novel topologies and control systems of 2-DOF LRM have been presented by various authors. Linear rotary actuator can be divided into several classes according to the stator number, namely, single stator structure, double stator structure, multi-stator structure and split-half stator structure [1]. The single stator structure is classified in several categories, including two sets of armature windings [2-4], concentrated windings [5][6] and spiral windings structure [7]. In [2-4], since the rotary and linear windings are orthogonal, there are crosscoupling in the magnetic field of the motor. In [5][6], there are multiple sets of concentrated windings. The control system and control strategy are difficult and complicated. In [7], the stator slot is spiral structure, and the eddy current loss is large. The double stator structure can be grouped into inner and outer double stator structure and side-by-side double stator structure [8-11], which has made the magnetic circuit of linear unit and rotary unit independently to achieve motion decoupling easily. However, the requirement of the manufacture and assemble are higher. Multiple stator structure can be classified into inductive structure and switched reluctance structure [12][13]. The stator consists of several independent stators, and the efficiency of the actuator is low and torque ripple is large. In addition, there are still lots of actuators, such as the actuator that only performs helical motion [14][15] has limited application.
The manufacture cost of an actuator with integrated magnetic gear function is high [16]. In [17-24], an actuator combining permanent magnet and magneto-resistive materials, LRM with hybrid excitation structure, step-type linear rotary reluctance actuator, micro-rotating linear actuators for micro endoscope catheters, rotary-linear voice coil motor, radial-gap helical ROTLIN machine, rotarylinear pneumatic actuator, and transverse-flux-type cylindrical linear synchronous motor are proposed by scholars at home and aboard, which can be used in special application situation. A linear rotary piezoelectric actuator including piezotranslator, friction block, sleeve, ring and pad was proposed, and the working principle, design and dynamic model of the actuator was analyzed in [25]. In [26], a piezoelectric actuator with a single cubic stator was presented, a through-hole and four piezoelectric elements bonded to the sides of the stator, which makes the actuator compact without any special manufacturing. The characteristics of cylindrical rotary-linear ultrasonic motor were analyzed, and the influence of exciting position and length of piezoelectric ceramic transducer plates on the performance of stator were researched and tested by the virtual instrument technology [27].

In order to meet the requirement of industrial application, the drive control system of 2-DOF LRM needs to not only detect the angular displacement, velocity, acceleration and torque/thrust, but also performs decoupling calculations. According to the literatures, the winding structure of the single winding LRM is similar to that of the traditional motor. The control strategy of the traditional motor can be 
adopted, but the motion decoupling and electromagnetic decoupling is difficult to perform, and the control strategy is complicated [28]. The two suits of independent windings can control linear and rotary motions, respectively. Then the actuator can be considered as two single winding motors. The control method and control strategy of the traditional motor can be adopted, but the actuator has motion decoupling problem. The cost of the control system increases [29][30]. According to the different requirements, the control system of multi-winding LRM consisting of rotation/linear speed closed loop, torque/thrust closed loop, linear position closed loop and current closed loop can be established, which can realize linear, rotary and spiral motions. However, there are many devices in the controller of the multi-winding LRM, the cost of the devices increases and the control circuit is complicated [31][32]. Therefore, the study of 2-DOF LRM has important research significance.

Since there is one set winding in the flux reversal linear rotary permanent magnet actuator (FR-LRPMA) used to realize the linear, rotary and spiral motions, solving the problems of the electromagnetic coupling and motion coupling is the key of the research. The decoupling process is analysed in detail in this paper. Firstly, the flux-linkage equation, voltage equation, torque equation and motion equation of FR-LRPMA are derived in the nine-phase stator coordinate system and dual $d q$-axis mover coordinate system. Secondly, an improved space vector pulse width modulation (SVPWM) is derived, and the control system is built by using modular and functional modeling methods in MATLAB/Simulink software. According to the function of the system to determine the composition of the various subsystems, the features of the subsystem are subdivided to determine the content of the next level subsystems, and the control model is built from top to bottom, which can achieve the linear, rotary and spiral movement. The simulink results at load condition are shown in section 3. Lastly, a prototype of FR-LRPMA and an experimental control platform are established. In order to verify the correctness of the improved control methods and strategies that can solve the problems of the electromagnetic and motion couplings, the control experiment of rotary, linear and spiral motions are conducted in section 4 .

\section{Control Model of FR-LRPMA}

\subsection{FR-LRPMA}

Fig. 1 shows the FR-LRPMA topology with three identical stator sections and two stator magnetic isolation aluminum rings in the axial direction. There are four sub-poles, namely, two permanent magnet (PM) poles and two ferromagnetic (Fe) poles, mounted on the surface of each stator pole. Eight mover salient poles in the circumferential direction are staggered by 22.5 degrees in the axial direction. The main circuit is dual-layer flux switching structure in circumferential and axial directions, which has better electromagnetic characteristics than single structure. Table 1 lists the main parameters of FR-LRPMA.

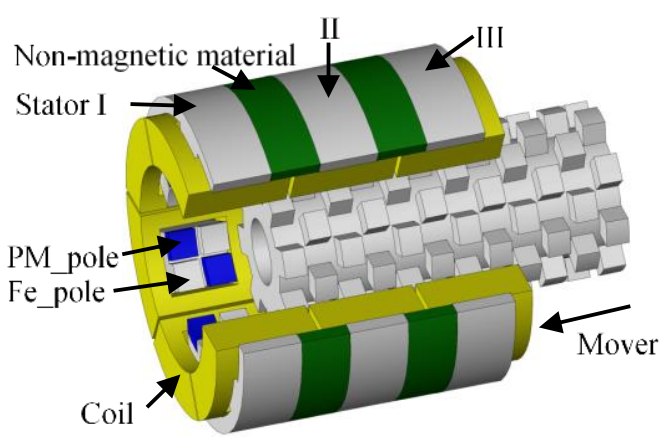

Fig. 1. The topology of FR-LRPMA.

Table 1. Main parameters of the FR-LRPMA

\begin{tabular}{|c|c|}
\hline Parameters & Value \\
\hline Mover diameter $(\mathrm{mm})$ & 60.2 \\
\hline PM height $(\mathrm{mm})$ & 5.2 \\
\hline Pole pitch $(\mathrm{z} / \mathrm{mm})$ & 21 \\
\hline Winding number & 80 \\
\hline Air-gap length $(\mathrm{mm})$ & 0.6 \\
\hline Stator pole width $(\mathrm{mm})$ & 21 \\
\hline PM width $(\theta / \mathrm{mm})$ & 9.9 \\
\hline PM width $(\mathrm{z} / \mathrm{mm})$ & 9.5 \\
\hline Stator yoke diameter $(\mathrm{mm})$ & 120 \\
\hline Move pole length $(\mathrm{z} / \mathrm{mm})$ & 7.5 \\
\hline Ratio of stator pole and slot $(\theta)$ & 1.2 \\
\hline Stator length $(\mathrm{z} / \mathrm{mm})$ & 97 \\
\hline Mover inner diameter $(\mathrm{mm})$ & 25 \\
\hline Coil diameter $(\mathrm{mm})$ & 0.6 \\
\hline Ratio of stator pole and slot $(\mathrm{z})$ & 1.5 \\
\hline Mover length $(\mathrm{z} / \mathrm{mm})$ & 144 \\
\hline
\end{tabular}

\subsection{The Control System Block Diagram}

Fig. 2 illustrates the control system block diagram of FRLRPMA. The control system consists of two speed loops, namely, the linear and rotary speed loops. The main components of the control system include the detection and speed calculation of linear and rotational positions, linear and rotational speeds, PI controller, dual $d q$ inverse transform, current sampling and SVPWM drive. Rotary motion feedback signal consists of rotary speed and rotary position signals. Linear motion feedback signal consists of linear speed and linear position signals.

$i_{d}=0$ is used in the servo drive system. Speed feedback is used to reflect the difference between the actual speed and the reference value of the drag system, and it can also make the actual speed close to the given speed by adjusting the PI parameters to improve the dynamic performance of the system. Current feedback is used to reflect the load condition, so that the torque component of the DC signal varies with the load, and it makes the working condition 
similar to that of DC motor.

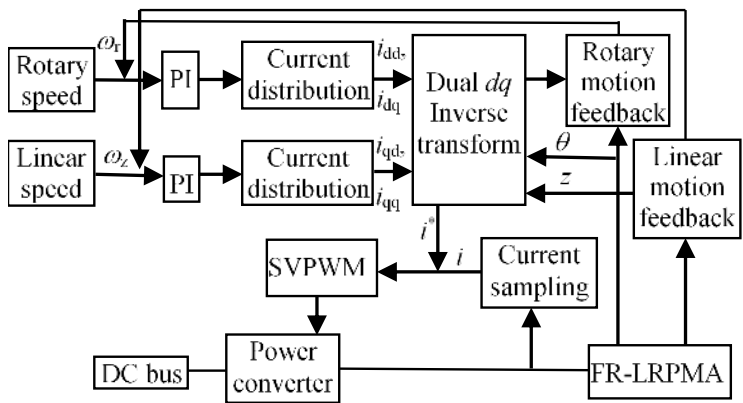

Fig. 2. The control system block diagram of FR-LRPMA.

The nine phase current signals of FR-LRPMA can be equivalent to the four DC current signals, shifted by 90 degrees to each other in the motion. The difference of the speed feedback value and the speed reference is taken as the input of the speed regulator. The current reference value is generated by the speed control, and the current reference value and the current feedback constitute a closed-loop current control by adjusting the PWM duty cycle to change the current value. Compared with the current reference value and the current feedback value, the output of the nine phase PWM is generated by SVPWM control. It is input into the established body model of FR-LRPMA, and then the output torque or thrust control is achieved.

\subsection{The mathematical model in the mover coordinate system}

In order to simplify the mathematic model of FR-LRPMA, the following assumptions are made: a) Motor parameters do not change with the working temperature, b) the hysteresis, saturation, and eddy current effects are ignored, c) sinusoidal distributions of the air-gap magnetic field are generated by the stator armature windings and the permanent magnets, d) the cogging effect, higher harmonics and the impact of magnetic flux leakage are ignored, and e) switching devices are ideal. f) The saturation of magnetic circuit is ignored.

The permanent magnet Back-EMFs of phase $m$ in linear motion and rotary motion are

$$
\begin{gathered}
E_{L p m_{-} m}=2 \pi p v_{z} N_{c} \phi_{p m} \cos \left(p_{\theta} \theta+\theta_{i}\right) \sin \left(2 \pi z / 3 \tau_{z}+z_{j}\right) / 3 \tau_{z} \\
E_{R p m_{-} m}=p \omega_{r} N_{c} \phi_{p m} \sin \left(p_{\theta} \theta+\theta_{i}\right) \cos \left(2 \pi z / 3 \tau_{z}+z_{j}\right)
\end{gathered}
$$

where $N_{c}$ is the number of windings, $\phi_{p m}$ is the amplitude of permanent magnet flux linkage in stator winding, $p_{\theta}$ is the pole pair in the rotary motion, $\tau_{z}$ is the pole pitch in the axial direction, $\omega_{r}$ is the mover rotational angular velocity, and $v_{z}$ is the linear speed of the mover.

The expression of the current in the nine phase stator windings is

$$
i_{m}=I_{\max } \cos \left(\omega_{\theta} t+\theta_{i}+\theta_{i \Delta}\right) \cos \left(\omega_{z} t+z_{j}+z_{j \Delta}\right)
$$

where $I_{\max }$ is the amplitude of the stator winding current, $\omega_{\theta}$ and $\omega_{z}$ are angular speeds in the circumferential and axial directions, $\theta_{i \Delta}$ and $z_{j \Delta}$ are the phase differences of flux linkage and the current in the circumferential and axial directions, respectively. $m$ is $A, B, C, D, E, F, G, H$ or $I$, respectively. $\theta_{i}$ and $z_{j}$ are the phase differences of different stator windings in the circumferential and axial directions, with values of $0,2 \pi / 3,-2 \pi / 3$, respectively.

Since the FR-LRPMA can achieve 2-DOF movement, a dual $d q$ mover coordinate system is established. Fig. 3(a) shows the winding arrangement. The linear, rotary or spiral motion is achieved by changing the phase of the current in the winding. Fig. 3(c) are the no load flux linkage waves, Phase $\mathrm{A}$ is taken as analysis objective. When the actuator is in rotary or linear motion, $(A, B, C),(A, D, G)$ have same sinusoidal PM linkage waveform, $(A, F),(A, H)$ and $(A, E, I)$ are the PM linkage waveforms of spiral motion. Fig. 3(b) is the dual $d q$ transformation. In the coordinate transformation of the motor, the $d d$ axis is taken to coincide with the stator A-phase winding. The $d d$ axis passes through the geometric center of the permanent magnet, the difference of electrical angle between the $d q$ and $q d$ axes and $d d$ axis is 90 electrical degrees both in the axial and circumferential directions, and the difference of electrical angle between the $q q$ and $d d$ axes is 90 electrical degrees both in the axial and circumferential directions.

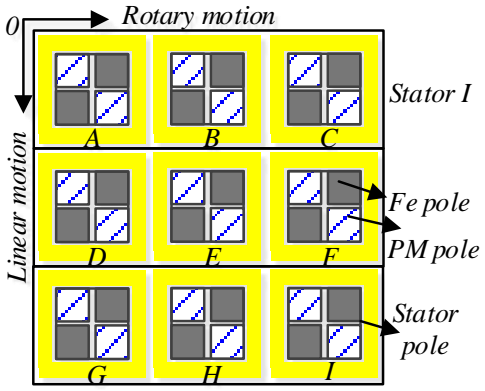

(a)

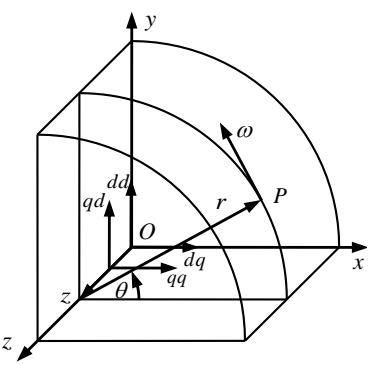

(b)

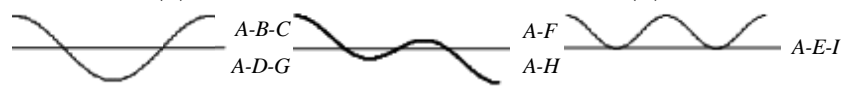

(c)

Fig. 3. FR-LRPMA winding arrangement and dual $d q$ transformation. (a) Winding arrangement, (b) Dual $d q$ transformation, (c) no-load flux linkage waves.

The current calculation is taken as an example, and the 0 phase is ignored, when it is in rotary motion,

$\left[\begin{array}{l}i_{A B C_{-} \mathrm{d}} \\ i_{A B C_{-} \mathrm{q}}\end{array}\right]=\sqrt{\frac{2}{3}} p(\theta)\left[\begin{array}{lll}i_{A} & i_{B} & i_{C}\end{array}\right]^{T},\left[\begin{array}{l}i_{D E F_{-} \mathrm{d}} \\ i_{D E F_{-} \mathrm{q}}\end{array}\right]=\sqrt{\frac{2}{3}} p(\theta)\left[\begin{array}{lll}i_{D} & i_{E} & i_{F}\end{array}\right]^{T}$, $\left[\begin{array}{l}i_{G H I_{-} \mathrm{d}} \\ i_{G H I_{-} \mathrm{q}}\end{array}\right]=\sqrt{\frac{2}{3}} p(\theta)\left[\begin{array}{lll}i_{G} & i_{H} & i_{I}\end{array}\right]^{T}$.

Then the expression is equivalent to

$$
\left[\begin{array}{lll}
i_{A B C_{-} d} & i_{D E F_{-} d} & i_{G H I_{-} d} \\
i_{A B C_{-} q} & i_{D E F_{-} q} & i_{G I_{-} q}
\end{array}\right]=\sqrt{\frac{2}{3}} p(\theta)\left[\begin{array}{ccc}
i_{A} & i_{D} & i_{G} \\
i_{B} & i_{E} & i_{H} \\
i_{C} & i_{F} & i_{I}
\end{array}\right]
$$

When the machine is in linear motion,

$$
\begin{aligned}
{\left[\begin{array}{ll}
i_{d d} & i_{d q} \\
i_{q d} & i_{q q}
\end{array}\right] } & =\sqrt{\frac{2}{3}} p\left(\theta_{z}\right)\left[\begin{array}{lll}
i_{A B C_{-} d} & i_{D E F_{-} d} & i_{G H I_{-} d} \\
i_{A B C_{-} q} & i_{D E F_{-} q} & i_{G H I_{-} q}
\end{array}\right]^{T} \\
\text { where } & p(\theta)=\left[\begin{array}{ccc}
\cos \theta & \cos (\theta-2 \pi / 3) & \cos (\theta+2 \pi / 3) \\
-\sin \theta & -\sin (\theta-2 \pi / 3) & -\sin (\theta+2 \pi / 3)
\end{array}\right] .
\end{aligned}
$$




\subsubsection{The flux equation}

The flux linkage equation in the mover coordinate system is

$$
\begin{aligned}
{\left[\begin{array}{ll}
\psi_{d d} & \psi_{d q} \\
\psi_{q d} & \psi_{q q}
\end{array}\right] } & =\frac{2}{3} P\left(\theta_{z}\right)\left[\begin{array}{lll}
\psi_{A} & \psi_{B} & \psi_{C} \\
\psi_{D} & \psi_{E} & \psi_{F} \\
\psi_{G} & \psi_{H} & \psi_{I}
\end{array}\right] P^{T}(\theta) \\
& =\left[\begin{array}{ll}
L_{d d} i_{d d} & L_{d q} i_{d q} \\
L_{q d} i_{q d} & L_{q q} i_{q q}
\end{array}\right]+\left[\begin{array}{ll}
\psi_{p m d d} & \psi_{p m d q} \\
\psi_{p m q d} & \psi_{p m q q}
\end{array}\right]
\end{aligned}
$$

where $L_{d d}, L_{d q}, L_{q d}$ and $L_{q q}$ are the inductances of $d d, d q$, $q d$ and $q q$ axes, respectively. Since the relative permeability of PM is approximately $1, L_{d d}=L_{d q}=L_{q d}=L_{q q}=9 L / 4, L$ is the inductance of single-phase stator winding. $\psi_{p m d d}, \psi_{p m d q}, \psi_{p m q d}$ and $\psi_{p m q q}$ are the PM flux components of the $d d, d q, q d$ and $q q$ axes, respectively. $\psi_{p m d d}=9 \psi_{p m d d} / 4, \psi_{p m d q}=\psi_{p m q d}=\psi_{p m q q}=0$.

\subsubsection{The voltage equation}

The expression of the voltage in the dual $d q$ mover coordinate system is

$$
\left\{\begin{array}{l}
u_{d d}=\frac{d \psi_{d d}}{d t}-\frac{P_{e q}}{2} K_{e q} \omega_{\theta} \psi_{d q}-\frac{P_{z q}}{2} K_{z q} \omega_{z} \psi_{q d}+R_{d d} i_{d d} \\
u_{d q}=\frac{d \psi_{d q}}{d t}+\frac{P_{e q}}{2} K_{e q} \omega_{\theta} \psi_{d d}-\frac{P_{z q}}{2} K_{z q} \omega_{z} \psi_{q q}+R_{d q} i_{d q} \\
u_{q d}=\frac{d \psi_{q d}}{d t}-\frac{P_{e q}}{2} K_{e q} \omega_{\theta} \psi_{q q}+\frac{P_{z q}}{2} K_{z q} \omega_{z} \psi_{d d}+R_{q d} i_{q d} \\
u_{q q}=\frac{d \psi_{q q}}{d t}+\frac{P_{e q}}{2} K_{e q} \omega_{\theta} \psi_{q d}+\frac{P_{z q}}{2} K_{z q} \omega_{z} \psi_{d q}+R_{q q} i_{q q}
\end{array}\right.
$$

where $\omega_{\theta}$ and $\omega_{z}$ are the electrical speeds of the mover in the circumferential and axial directions, respectively. $\omega_{z}=2 \pi v_{\mathrm{z}} / \tau_{\mathrm{z}}, R_{d d}, R_{d q}, R_{q d}$ and $R_{q q}$ are the resistances of $d d, d q$, $q d$ and $q q$ axes, respectively. $R_{d d}=R_{d q}=R_{q d}=R_{q q}=9 R / 4$.

According to (7), the expression is

$$
\left\{\begin{aligned}
d i_{d d} / d t & =\left(u_{d d}-R_{d d} i_{d d}\right) / L+P_{e q} K_{e q} \omega_{\theta} i_{d q} / 2+P_{z q} K_{z q} \omega_{z} i_{q d} / 2 \\
d i_{d q} / d t & =\left(u_{d q}-P_{e q} K_{e q} \omega_{\theta} \psi{ }_{p m d d} / 2-R_{d q} i_{d q}\right) / 2 \\
& -P_{e q} K_{e q} \omega_{\theta} i_{d d} / 2+P_{z q} K_{z q} \omega_{z} i_{q q} / 2 \\
d i_{q d} / d t & =\left(u_{q d}-P_{z q} K_{z q} \pi v_{z} \psi_{p m d d} / \tau_{z}-R_{q d} i_{q d}\right) / L \\
& +P_{e q} K_{e q} \omega_{\theta} i_{q q} / 2-P_{z q} K_{z q} \omega_{z} i_{d d} / 2 \\
d i_{q q} / d t & =\left(u_{q q}-R_{q q} i_{q q}\right) / L-P_{e q} K_{e q} \omega_{\theta} i_{q d} / 2-P_{z q} K_{z q} \omega_{z} i_{d q} / 2
\end{aligned}\right.
$$

According to the voltage differential equation (8), the current sub-model (FR-LRPMA source) is established in the mover coordinate system, including the current and voltage sub-models of $d d, d q, q d$ and $q q$ axes. In the sub-model, the current signals are given in the nine-phase stator coordinate system according to the nine-phase voltage signals, the mover linear position, rotation speed and rotation position information. The sub-model consists of the sub-model of nine-phase to four-phase (dual $d q$ ), the current and voltage sub-model in the mover coordinate system $\left(U_{d d q q}-i_{d d q q}\right)$, and the sub-model of four-phase to nine-phase (anti-dual $d q$ ). The voltage sub-model of nine-phase conversion to fourphase is achieved by the voltage conversion from nine-phase stator coordinate system to dual $d q$-axis mover coordinate system. The current sub-model of four-phase conversion to nine-phase is built by the current conversion from dual $d q$ axis mover coordinate system to nine-phase stator coordinate system.

The expression of the torque in the circumferential direction is

$$
T_{e}=\frac{9 P_{e q}}{8} K_{e q}\left[-i_{d q} \psi_{p m}-i_{d q} i_{d d}\left(L_{d d}-L_{d q}\right)-i_{q q} i_{q d}\left(L_{q d}-L_{q q}\right)\right]
$$

The expression of the thrust in the axial direction is

$$
F_{e}=9 \pi P_{z q} K_{z q}\left(-i_{q d} \psi_{d d}-i_{q q} \psi_{d q}+i_{d d} \psi_{q d}+i_{d q} \psi_{q q}\right) / 4 \tau_{z}
$$

According to (9) and (10), the torque sub-model and the force sub-model are built.

\subsubsection{The mechanical equation}

According to Newton's law of motion, without considering the torsional elastic torque coefficient of the conditions, the mechanical equations of FR-LRPMA in the circumferential and axial directions are

$$
\begin{aligned}
& T_{e}=J_{m} d \omega_{r} / d t+P_{e q} K_{e q} B_{f r} \omega_{r} / 2+T_{l} \\
& F_{e}=m_{m} d v_{z} / d t+P_{z q} K_{z q} B_{f z} v_{z} / 2+F_{l}
\end{aligned}
$$

where $J_{m}$ and $m_{m}$ are the inertia and mass of the actuator mover, respectively. $\omega_{r}=d \theta_{r} / d t, B_{f r}$ and $B_{f z}$ are the system friction coefficients of rotation and linear movement, respectively. $T_{l}$ and $F_{l}$ are the cogging torque and linear detent force in the rotary and linear motion, respectively.

The mechanical loss is ignored. According to (11) and (12), the rotational sub-model and the linear sub-model are established. The input and output signals of linear motion sub-model are the linear electromagnetic force and the linear mechanical speed, respectively. The input and output quantity signals of rotary motion sub-model are the torque and mechanical angular speed, respectively. The sub-model of linear and rotary motions can give the different load types of the actuator by selecting switch model. The enabled subsystem is the subsystem of each simulation step executed when the control signal is positive. And it can avoid the operation when the rotary speed value is zero. Then the constant power load torque operation can be achieved.

\subsubsection{The torque and thrust equation}

According to the electromechanical energy conversion principle, the magnetic total energy $W_{m}^{\prime}(\theta, z, i)$ is the integration of the flux and the current, and the expressions of torque and linear thrust are

$$
\left\{\begin{array}{l}
T_{e}=-p_{\theta} N \phi_{\max } \sum \sum i_{n m} \sin \left(\theta+\theta_{i}\right) \cos \left(\eta+z_{j}\right) \\
F_{e}=-2 \pi N \phi_{\max } \sum \sum i_{n m} \cos \left(\theta+\theta_{i}\right) \sin \left(\eta+z_{j}\right) / \tau_{z}
\end{array}\right.
$$

where $\theta=p_{\theta} \theta_{\text {mec }}, \quad \eta=2 \pi \mathrm{z} / \tau, N$ is the number of the winding. $\phi_{\max }$ is the maximum flux linkage of the stator pole. $\theta_{i}$ and $z_{j}$ are the phase differences between the different stator teeth, respectively.

When $i_{d d}=i_{q q}=0$, the expressions of the torque and force are

$$
\left\{\begin{array}{l}
T_{e}=-9 P_{e q} K_{e q} i_{d q} \psi_{p m \text { max }} / 8 \\
F_{e}=-9 \pi P_{z q} K_{z q} i_{q d} \psi_{p m \text { max }} / 4
\end{array}\right.
$$

The sub-models of torque and thrust of the actuator are 
built according to (14). When $i_{d d}=i_{q q}=0$, the torque is related to the $d q$ axis current component $i_{d q}$, and the thrust is related to the $q d$ axis current component $i_{q d}$. Then the relationship between the torque and the $d q$ axis current is linear, and the relationship between the thrust and the $q d$ axis current is also linear. In the actual control system, the position of the $d d$ axis is detected, the stator nine-phase winding magnetomotive force after dual $d q$ transform only contains the $d q$ and $q d$ axes components, and then the rotary, linear or spiral movement of FR-LRPMA is achieved.

\subsection{Improved SVPWM}

Since the movement range of the mover is only $47 \mathrm{~mm}$ in the axial direction, the rotary motion is the main motion type and the linear motion is used to change the linear position. Since the linear speed is about $4 \mathrm{~mm} / \mathrm{s}$, the actuator can not work at low speed by the traditional SVPWM. Then the improved SVPWM is proposed in the paper, which combines SVPWM and PWM modulation technology. When the actuator is in rotary motion, assuming that $i_{A a}=I_{\max } \cos \left(\theta+\theta_{\Delta}\right) \cos \left(\theta^{\prime}+\theta_{\Delta}^{\prime}\right)$, the waveform of BackEMF is symmetrical. Then $T_{e}=-9 I_{\max } \psi_{p m} \sin \theta_{\Delta} \cos \theta_{\Delta}^{\prime} / 4$, similarly, $\quad F_{e}=-9 \pi I_{\max } \psi_{p m} \cos \theta_{\Delta} \sin \theta_{\Delta}^{\prime} / 2 \tau$. If $\theta_{\Delta}^{\prime}=0, T_{e}=-9 I_{\max } \psi_{p m} / 4$. If $\theta_{\Delta} \neq 90^{\circ}$ and $\theta_{\Delta}^{\prime} \neq 0$, then $F_{e}=-9 \pi I_{\max } \psi_{p m} \cos \theta_{\Delta} \sin \theta_{\Delta}^{\prime} / 2 \tau \neq 0, \quad$ the linear position can change. Since the rotary, linear or spiral motion are achieved by one set of winding, then table 2 is the correspond relationship of switch time and sector when in rotary or linear motion. Table 3 is the switching time. The definition of the variables is

$$
\left\{\begin{array}{l}
B_{0}=U_{\beta} \\
B_{1}=\sqrt{3} U_{\alpha}-U_{\beta} \\
B_{2}=-\sqrt{3} U_{\alpha}-U_{\beta} \\
N=4 \operatorname{sign}\left(B_{2}\right)+2 \operatorname{sign}\left(B_{1}\right)+\operatorname{sign}\left(B_{0}\right)
\end{array}\right.
$$

Then the switching time is calculated as

$$
\left\{\begin{array}{l}
T_{a}=\left(T-T_{x}-T_{y}\right) / 4 \\
T_{b}=T_{a}+T_{x} / 2 \\
T_{c}=T_{b}+T_{y} / 2
\end{array}\right.
$$

Table 2. The correspond relationship of switch time and sector when in rotary or liner motion

\begin{tabular}{|c|c|c|c|c|c|c|}
\hline Sector & I & II & III & IV & V & VI \\
\hline$T_{c m 1}$ & $t_{1}$ & $t_{2}$ & $t_{3}$ & $t_{3}$ & $t_{2}$ & $t_{1}$ \\
\hline$T_{c m 2}$ & $t_{2}$ & $t_{1}$ & $t_{1}$ & $t_{2}$ & $t_{3}$ & $t_{3}$ \\
\hline$T_{c m 3}$ & $t_{3}$ & $t_{3}$ & $t_{2}$ & $t_{1}$ & $t_{1}$ & $t_{2}$ \\
\hline
\end{tabular}

where $t_{1}=\left(t-t_{-} 1-t_{-} 2\right) / 2, t_{2}=t_{1}+t_{-} 1, t_{3}=t_{2}+t_{-} 1$.

Table 3. The correspond relationship of switch time and sector when in rotary, linear and spiral motion

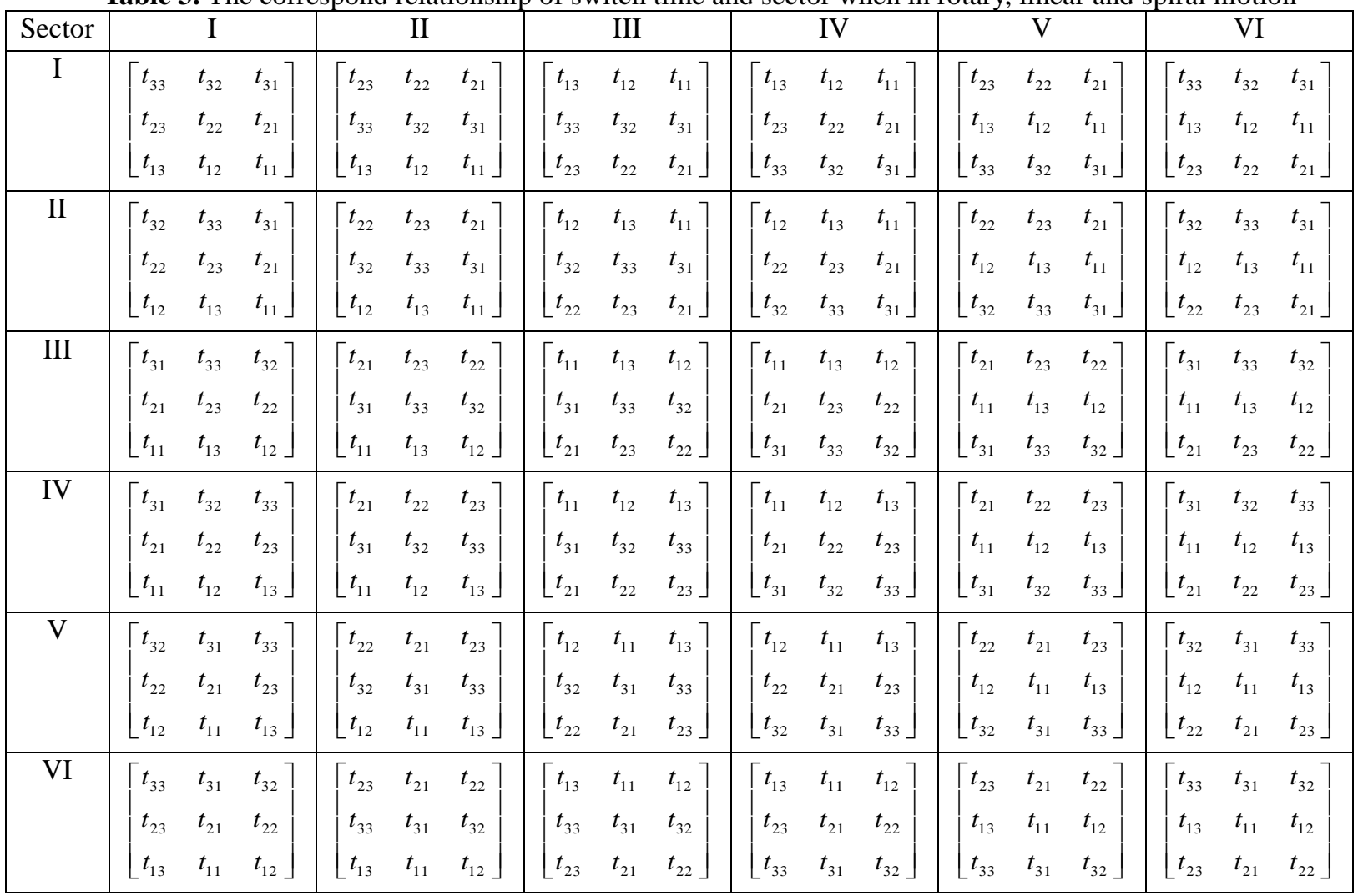

where $t_{11}=\left(t_{T}-t_{T_{-} 11}-t_{T_{-} 12}\right) / 2, \quad t_{12}=t_{T 11}+t_{T_{-} 11}, \quad t_{13}=t_{T 12}+t_{T_{-} 11}, \quad t_{21}=\left(t_{T}-t_{T_{-} 21}-t_{T_{-} 22}\right) / 2 \quad, \quad t_{22}=t_{T_{21}}+t_{T_{-} 21} \quad, \quad t_{23}=t_{T 22}+t_{T_{-} 21}$. $X=u_{\beta}, Y=\left(\sqrt{3} u_{\alpha}+u_{\beta}\right) / 2, \quad Z=\left(-\sqrt{3} u_{\alpha}+u_{\beta}\right) / 2, \quad t_{T_{-} 11}=t_{-} 1 \cdot T_{-} 1, \quad t_{T_{-} 12}=t_{-} 1 \cdot T_{-} 2, t_{T_{-} 21}=t_{-} 2 \cdot T_{-} 1, \quad t_{T_{-} 22}=t_{-} 2 \cdot T_{-} 2 . t t_{-} 1, t_{-} 2$ is the action time of adjacent non-zero vector when in rotary motion, respectively. $T_{-} 1, T_{-} 2$ are the action time of adjacent non-zero vector when in linear motion, respectively. 


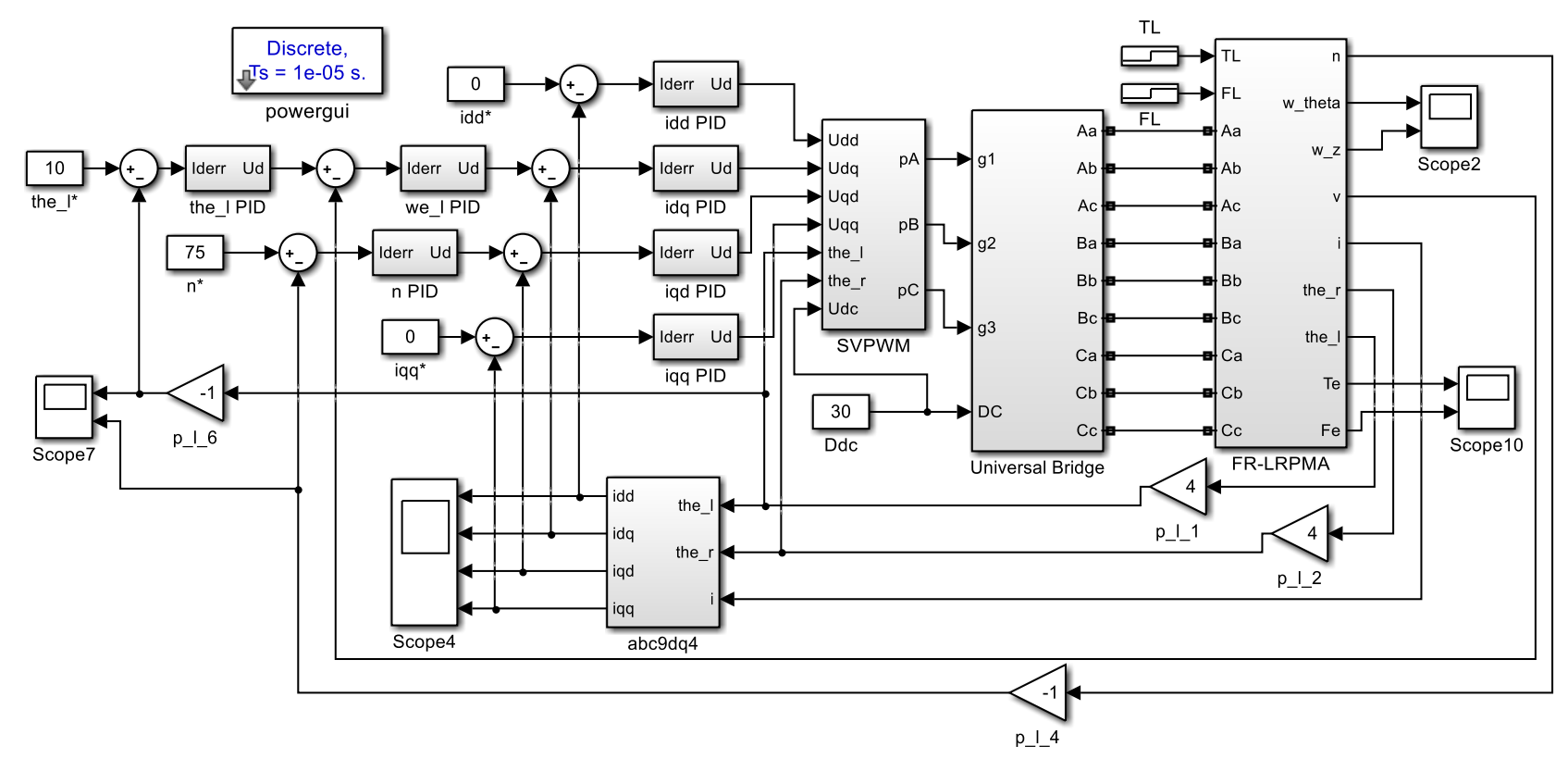

Fig. 4. The overall block diagram of FR-LRPMA control system simulation model.

\subsection{The simulation model}

The control strategy of FR-LRPMA combines the control strategy of flux reversal machine and LRPMA. Fig. 4 is the overall block diagram of FR-LRPMA control system simulation model. In order to verify the correctness of mathematical model and control strategy, the FR-LRPMA subsystem and improved SVPWM control module are built using the general Simulink module. Power converter subsystem is built using the PSB module, and the connection of several control system simulation model is constituted by the application interface module port design.

Table 4 is the main parameters of simulation system of FR-LRPMA. Fig. 5 are the cogging torque and linear detent force waveforms. The voltage measurement sub-model is established according to the current sub-model. The submodels of linear and rotary position signals are established according to the requirement of the improved SVPWM. The input signal of FR-LRPMA sub-system is the voltage of the nine-phase half-bridge module. The output signals of FR-LRPMA sub-system are nine-phase current signals, and the position signals of mover in the axial and circumferential directions. The other parameters need to be observed.

Table 4. The main parameters of simulation system of FR-LRPMA

\begin{tabular}{|c|c|}
\hline Parameters & Value \\
\hline Rated power & $400 \mathrm{~W}$ \\
\hline Rated speed & $1500 \mathrm{rpm}$ \\
\hline Rated current & $5 \mathrm{~A}$ \\
\hline Rated voltage & $30 \mathrm{~V}$ \\
\hline Rated frequency & $200 \mathrm{~Hz}$ \\
\hline Stator resistance & $1.2 \Omega$ \\
\hline Stator inductance & $2.47 \mathrm{mH}$ \\
\hline Linear motion distance & $47 \mathrm{~mm}$ \\
\hline
\end{tabular}

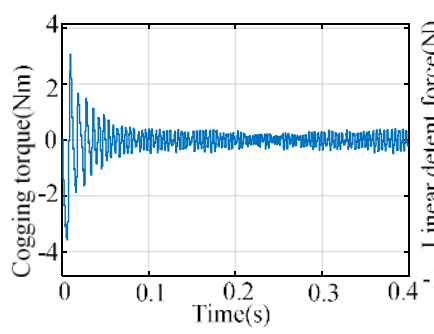

(a)

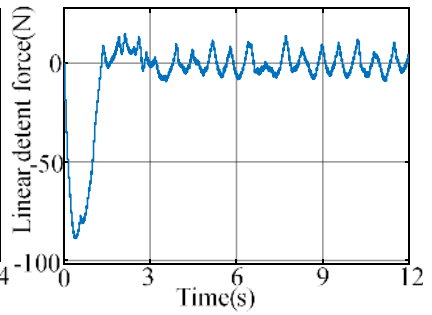

(b)
Fig. 5. Cogging torque and linear detent force waveforms.(a) Cogging torque, (b) Linear detent force.

\section{Load Simulation Analysis of FR-LRPMA}

\subsection{Rotation Motion}
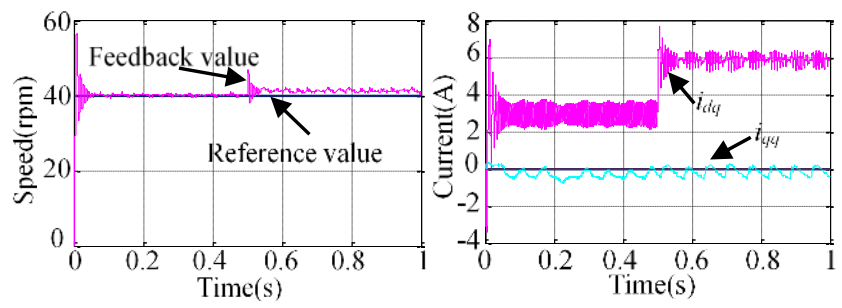

(a)

(b)

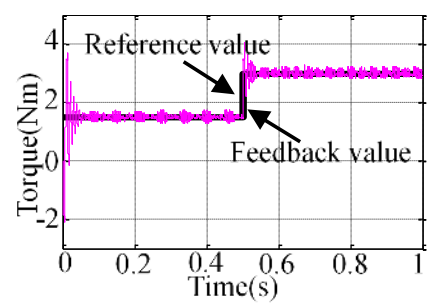

(c)

Fig. 6. Variable waveforms of rotary regulation speed motion in the load condition.(a) Rotary speed, (b) Four phase currents in the mover coordinate system, (c) Rotary torque.

When the rotary speed is $40 \mathrm{rpm}$, the current phase of stator winding keeps constant in the axial direction, and 
phase $A$, phase $B$ and phase $C$ of three independent stators are at $0^{\circ}, 120^{\circ}$ and $240^{\circ}$, respectively. Fig. 6 (a) shows the speed waveform, Fig. 6 (b) the four-phase current component waveforms in the mover coordinate system, and Fig. 6 (c) the torque waveform. When the actuator is operated at $0.5 \mathrm{~s}$, the amplitude of the current value changes from $3 \mathrm{~A}$ to $6 \mathrm{~A}$, then the torque changes from $1.5 \mathrm{Nm}$ to 3 $\mathrm{Nm}$. It is clear that the torque is proportional to the $d q$ axis current component. The ripple of the speed, the torque and the $d q$ axis current component are larger than those in other stage when the rotary speed changes.

\subsection{Linear Motion}

When the linear speed is $3 \mathrm{~mm} / \mathrm{s}$, the current phase of the stator winding remains constant in the circumferential direction. The phases of the phase $A$, phase $B$ and phase $C$ of three independent stators are $0^{\circ}, 120^{\circ}$ and $240^{\circ}$, respectively. Fig. 7 (a) shows the linear regulation waveform, Fig. 7 (b) the four-phase current component waveforms in the mover coordinate system, and Fig. 7 (c) the thrust waveform. At $0.5 \mathrm{~s}$, the amplitude value of the current changes from $2 \mathrm{~A}$ to $4 \mathrm{~A}$, then the torque changes from $2 \mathrm{~N}$ to $4 \mathrm{~N}$. It is clear that the thrust is proportional to the $q d$-axis current component.

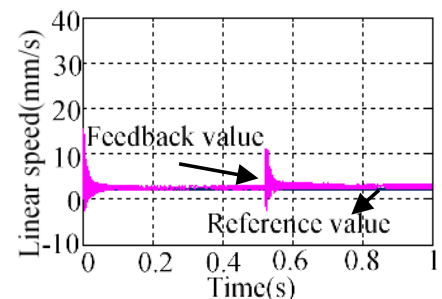

(a)

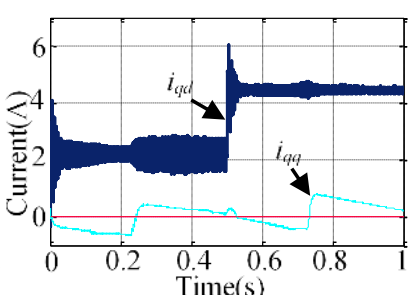

(b)

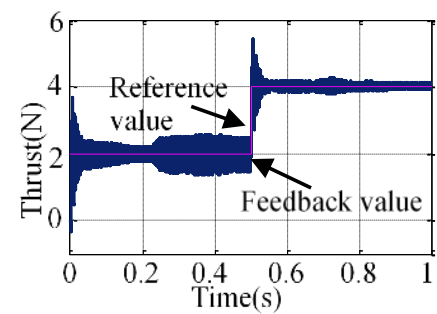

(c)

Fig. 7. Variable waveforms of linear regulation speed motion in the load condition.(a) Linear speed, (b) Four phase currents in mover coordinate system, (c) Thrust.

\subsection{Spiral Motion}

Fig. 8 (a) shows the rotary and linear speeds, Fig. 8 (b) he four phase currents in the mover coordinate system, and Fig. 8 (c) the torque and the thrust waveforms. When the rotary speed is $40 \mathrm{rpm}$ and the linear speed is $3 \mathrm{~mm} / \mathrm{s}$, the amplitude value of the current $i_{d q}$ changes from $3 \mathrm{~A}$ to $6 \mathrm{~A}$ at $0.5 \mathrm{~s}$. The amplitude value of the current $i_{q d}$ changes from $2 \mathrm{~A}$ to $4 \mathrm{~A}$ at $1 \mathrm{~s}$, then the torque changes from $1.5 \mathrm{Nm}$ to $3 \mathrm{Nm}$, and the thrust changes from $2 \mathrm{~N}$ to 4 $\mathrm{N}$. It is clear that the rotary and linear motions are independent to each other.

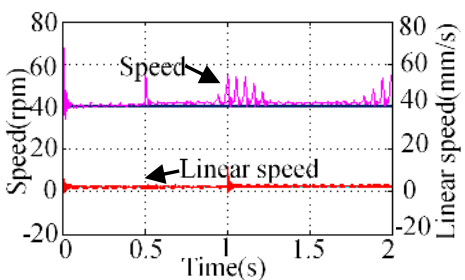

(a)

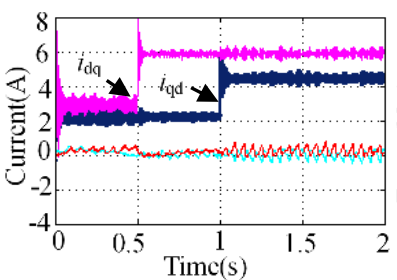

(b)

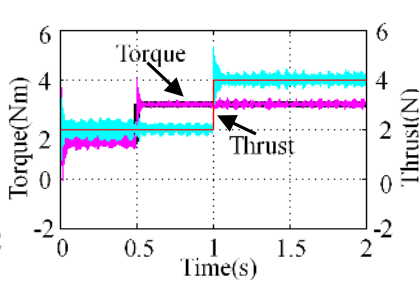

(c)
Fig. 8. Variable waveforms of spiral regulation speed motion in the load condition.(a) Rotary and linear speeds, (b) Four phase currents in the mover coordinate system, (c) Torque and thrust.

\section{Experimental Results and Discussions}

\subsection{Types of Graphics}

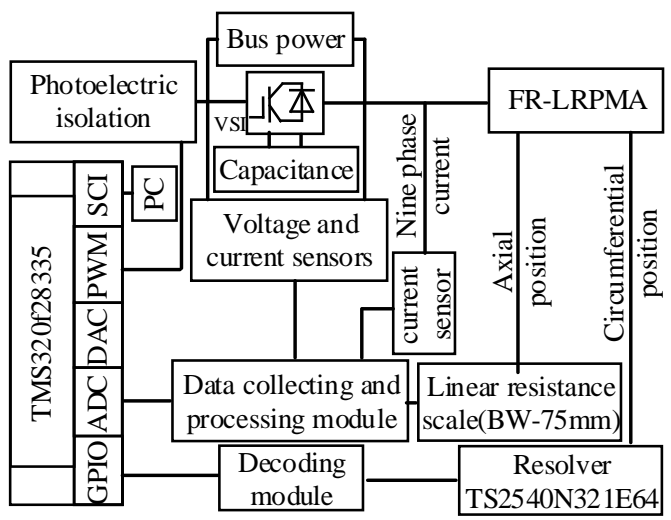

Fig. 9. The configuration of the experimental control system of FR-LRPMA.

Fig. 9 shows the configuration of the control system of FR-LRPMA based on TMS320F28335. The control system of FR-LRPMA consists of the DSP controller, data collecting and processing module, drive module, which can realize the control algorithm, sample and filtering of th e analog signals of currents and voltages, axial positio $\mathrm{n}$ signal, the digital signal of the rotary speed from th e resolver. The control system communicates with the host computer by the serial communication. The analogue 
sampling period is $100 \mu$ s and the actual computation time of the algorithm is about $30 \mu \mathrm{s}$ in the DSP. The inverter bridge consists of eighteen metal-oxide-semiconductor field-effect transistors (IRFP 460) with a rating of 20A, $500 \mathrm{~V}$, and it makes the whole drive circuit simpler and more reliable. TLP250 is taken as optocoupler device. The measure range of the voltage measured hall voltage sensor (VSM025A) is $10 \sim 500 \mathrm{~V}$. The output voltage signal of the hall current sensor (CSM006NPT3.3) is 0-3.3V. A high precision linear resistance scale (BW-75mm) is selected to measure axial displacement, and its measure range and measure accuracy are $75 \mathrm{~mm}, 5 \mu \mathrm{m}$, respectively. The circumferential position and rotary speed are measured by the resolver (TS2540N321E64), and its excitation frequency is $10 \mathrm{kHz}$.

\subsection{Experimental Platform}

Fig. 10 illustrates the experiment platform of FRLRPMA. Fig. 10 (a) shows the test platform of the torque and cogging torque, which consists of the prototype, the control system and the torque measuring instrument (MT6425). Fig. 10 (b) shows the hardware of the control system, which consists of four parts: signal acquisition module, drive module, decoding module and DSP control board. Fig 10 (c) shows the test platform of electromagnetic force in the axial direction, which contains the prototype, the S-type tension sensor and the operational amplifier. When the prototype is in linear motion, the mover is connected to the linear motor by the S-type tension sensor, which is fixed to the pedestal.

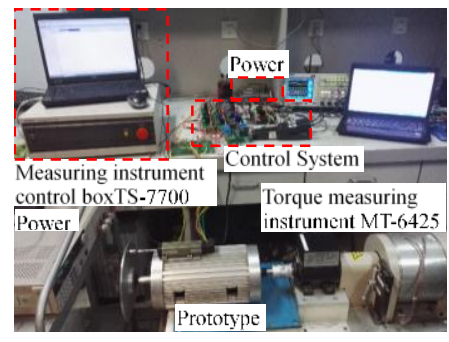

(a)

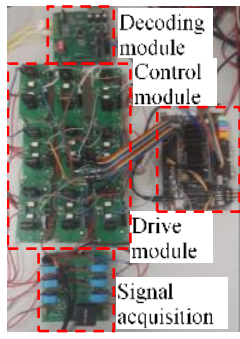

(b)

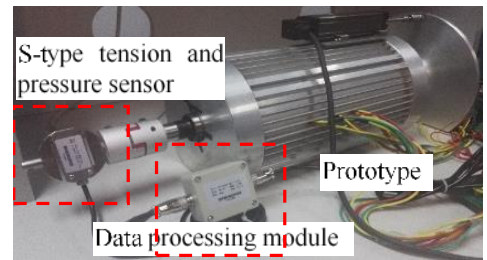

(c)

Fig. 10. The experiment platform of FR-LRPMA.(a) Testing of electromagnetic torque, (b) The control system, (c) Testing of electromagnetic force.

\subsection{The Control Experiment of Rotary, Linear and Spiral Motions}

The optimization of cogging torque and linear detent force is an important part in the design. In order to verify the optimization results, it is needed to measure the cogging torque in different linear positions and the linear detent force in different mover angles in the test experiment.

Fig. 11 plots the experimental waveforms of rotary motion of FR-LRPMA, (CH1:2V, $\mathrm{CH} 2: 2 \mathrm{~V}, \mathrm{CH} 3: 2 \mathrm{~A}$, $\mathrm{CH} 4: 2 \mathrm{~A}, \mathrm{M}: 50 \mathrm{~ms}$ ) when the speed is $150 \mathrm{rpm}$ using improved SVPWM. 1 indicates the current waveform of the phase A in Fig. 11(a), and shows the linear position signal in Fig. 11(b). 2 is the position signal of the resolver, and 3 and 4 indicate the current waveforms of phase A, phase B, respectively. It is clear that the prototype design and the control model are correct. The slope of signal 2 from the top point to the bottom point is stable, which is verified that the rotary speed is stable. The current value of phase A in Fig. 11(1) is digital to analog signal generated by the DSP controller, which is similar to the actual current signal of phase A in Fig. 11(3), it is clear that the tracking and load regulation response is fast. Fig. 12(a) shows the measurement results of the linear motion $(\mathrm{CH} 1: 2 \mathrm{~V}, \mathrm{CH} 2: 1 \mathrm{~V}$ $\mathrm{CH} 3: 5 \mathrm{~A}, \mathrm{CH} 4: 5 \mathrm{~A}, \mathrm{M}: 2.5 \mathrm{~s})$. The armature magnetic field can be obtained by changing the phase of the nine phase currents, then we can obtain the magnetic field for spiral motion. Fig. 12(b) is the experiment result of the spiral motion. 1 indicates the linear position signal, 2 is the rotary angular position signal, and 3 and 4 indicate the current waveforms of phase A and phase B, respectively. The linear position signal $(0-47 \mathrm{~mm})$ is measured by linear resistance ruler, which is converted to voltage signal $(0-3.2 \mathrm{~V})$, the rotary position signal $(0-2 \pi)$ is measured by resolver, which is converted to voltage signal $(0-2.4 \mathrm{~V})$, and then the voltage signal can reflect the control accuracy.

In Fig. 11 and Fig. 12, it is clear that there is no linear displacement when the actuator is in rotary motion and there is no rotary displacement when the actuator is in linear motion. The rotary motion and linear motion can work independently. Linear and rotary speed frequencies are at the lowest value $0.1 \mathrm{~Hz}$ as shown in Fig. 12(b), and the linear position and rotary position are changing simultaneously. It is concluded that the control algorithm and the control strategy are correct and effective. Spiral motion is a combination of rotation motion and linear motion, and the spiral motion frequency can be composed by the rotary and linear motion frequencies. The rotary speed of spiral motion can be higher with the rotary frequency increasing. Since the effective magnetic circuits are different, the amplitudes of phase A and phase B are 
slightly different as shown by 3 and 4 in Fig. 12(b).

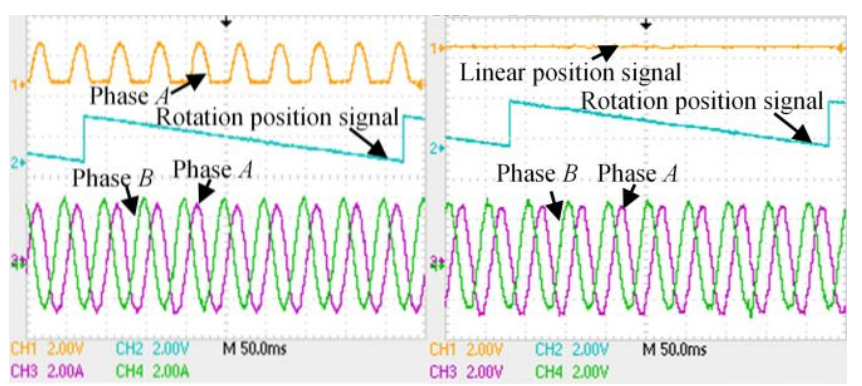

(a)

(b)

Fig. 11. The rotary motion of FR-LRPMA.(a) Feedback signal verification, (b) Operating status.

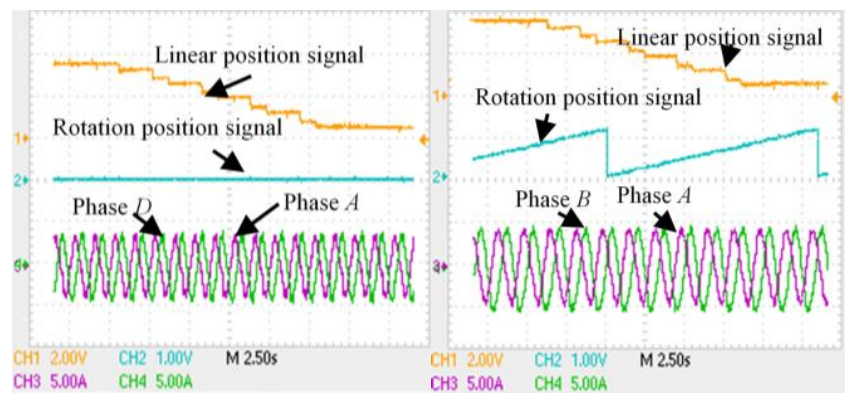

(a)

(b)

Fig. 12. Experimental results.(a) Linear motion, (b) Spiral motion.

\subsection{The Experiment Results of Electromagnetic Characteristics}

Fig. 13 shows the cogging torque test results in different linear positions: $\mathrm{L}=0 \mathrm{~mm}$ and $\mathrm{L}=20 \mathrm{~mm}$. The rotary motion is steady without axial movement in different linear position. The mover shaft of the actuator and the torque tester are fixed through the coupler. According to the different linear position, the voltage phases of the phase A, phase $B$ and phase $C$ are different. When $L=0 \mathrm{~mm}$, the voltage phase difference of phase A and phase $\mathrm{D}$ is $180^{\circ}$ in the axial direction, and the voltage phase difference of phase $\mathrm{A}$, phase $\mathrm{B}$ and phase $\mathrm{C}$ is $120^{\circ}$ in the circumferential direction, then the cogging torque can be measured. When $\mathrm{L}=20 \mathrm{~mm}$, the cogging torque can be tested using the same method. It is clear that the amplitudes of the cogging torque in different linear positions are the same, and the amplitude in the same linear position remains steady. The cogging torque amplitude of the experiment result is consistent with that of the simulation as shown in Fig. 5(a).

The tension sensor is fixed on the mover shaft, Fig. 14 shows the test result of the linear detent force when the angular angle $\theta=0^{\circ}$ and $\theta=15^{\circ}$. It is clear that the amplitudes of the linear detent force under different angular positions are the same, and the amplitude in the same angular position is constant. The linear detent force amplitude of the experiment result is consistent with that of the simulation as shown in Fig. 5(b). Fig. 15 shows the torque waveform under the condition with bus voltage of 5 $\mathrm{V}$, and the load is $1.06 \mathrm{Nm}$. Fig. 15(a) shows the torque waveform at $166 \mathrm{rpm}$. Fig. 15(b) is the rotary speed waveform with no fluctuation. It is clear that FR-LRPMA can operate stably under the load condition.

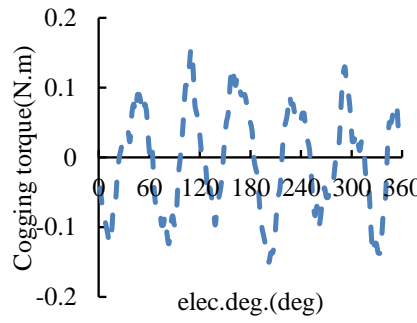

(a)

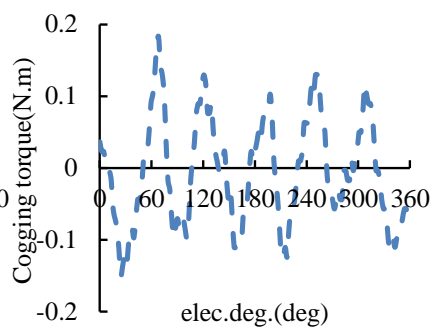

(b)
Fig. 13. The cogging torque test results in different axial linear positions.(a) Cogging torque when the linear position is at $0 \mathrm{~mm}$, (b) Cogging torque when the linear position is at $20 \mathrm{~mm}$.

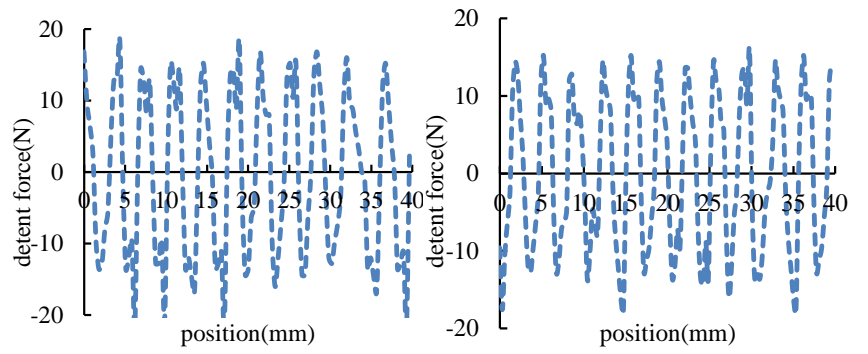

(a)

(b)

Fig. 14. Linear detent force under different angle positions. (a) $\theta=0^{\circ}$, (b) $\theta=15^{\circ}$.

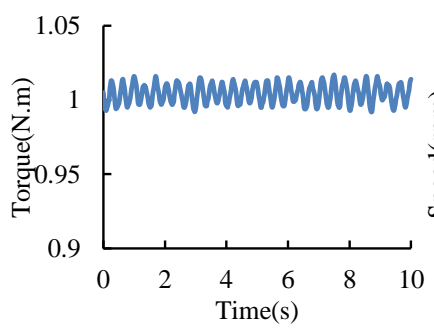

(a)

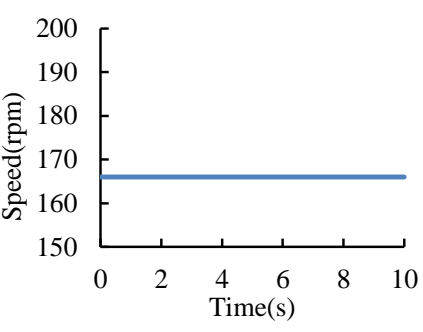

(b)
Fig. 15. The torque test result.(a) Torque waveform (166 rpm, $1.06 \mathrm{Nm}$ ), (b) Speed waveform.

Fig. 16(a) is the torque waveform when the rotary speed is $900 \mathrm{rpm}$, the current is $5 \mathrm{~A}$. Compared with the torque result of Fig. 15(a), the torque ripple is higher. It can be concluded that the closed-loop system is more stable when the actuator works at load $1.06 \mathrm{~N}$. m than that when the load torque is 4.75 N. $m$ in Fig. 16(a). Fig. 16(b) is the torque-speed map. It can be seen that the actuator can 
achieve the optional characteristics at different speed and torque conditions. The high-efficiency work areas are concentrated and the control system is stable.

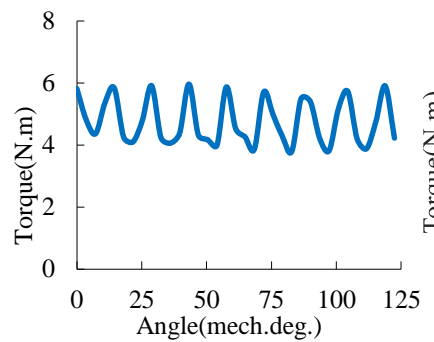

(a)

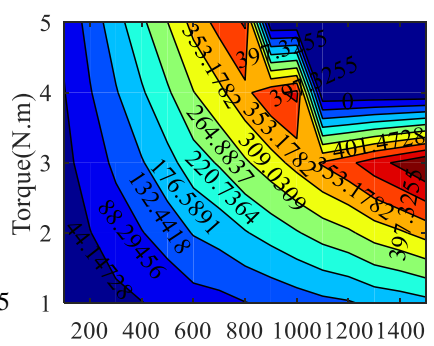

(b)
Fig. 16. The torque waveform at $1500 \mathrm{rpm}$. (a) Torque waveform, (b) Torque-speed map.

\section{Conclusions}

The electromagnetic and motion decoupled process of FR-LRPMA is detailed described in the paper. The control equations of FR-LRPMA are derived in nine phase stator and dual dq-axis mover coordinate systems, and its mathematical model and the control model in the mover coordinate system are established by the improved SVPWM in MATLAB/Simulink software, then the rotary, linear or spiral motion are achieved. A prototype of FRLRPMA is made and an experimental platform of FRLRPMA is set up. The waveforms of torque/cogging torque in different linear positions and the linear detent force in different angular positions are reported, which are consistent with the results obtained from the simulation. And it can also verify that the prototype optimization, mathematical model, control model and control strategy are correct.

\section{Acknowledgements}

This work was supported by the Natural Science Foundation of China under Grant 51905003, Natural Science Foundation of Anhui Province under Grant 1908085QE207, China Postdoctoral Science Foundation under Grant 2019M652161 and Young Teacher Natural Science Foundation of AUST under Grant QN2018107.

\section{References}

[1] J. Si, L. Xie, and X. Xu, et al., "Static coupling effect of a two-degree-of-freedom direct drive induction motor," IET Electric Power Applications, vol. 11, no. 4, pp. 532-539, Apr. 2017.
[2] K. Kluszczynski and M. Szczygiel, "How to convert a factory-manufactured induction motor into rotarylinear motor? part 1 constructional issues," in Proc. 15th REM, Oct. 2014, pp. 1-6.

[3] K. J. Meessen, J. J. H. Paulides and E. A. Lomonova, "Analysis of a novel magnetization pattern for 2-DOF rotary-linear actuators," IEEE Trans. Magn., vol. 48, no. 11, pp. 3867-3870, Oct. 2017.

[4] E. Amiri, "Circuit modeling of double-armature rotarylinear induction motor," in Proc. 2014-40th IECON, Nov. 2014, pp. 431-436.

[5] P. Jin, H. Lin, and S. Fang, et al., "3-D analytical linear force and rotary torque analysis of linear and rotary permanent magnet actuator," IEEE Trans. Magn., vol. 49, no. 7, pp. 3989-3992, Jul. 2013.

[6] S. Miri' c, A. Tüysüz and J. W. Kolar, "Comparative evaluation of linear-rotary actuator topologies for highly dynamic applications," in Proc. 2017 IEMDC, Aug. 2017, pp. 1-7.

[7] S. Tanaka, T. Shimono and Y. Fujimoto, "Optimal design of length factor for cross-coupled 2-DOF motor with Halbach magnet array," in Proc. 2015 ICM, Apr. 2015, pp. 529-534.

[8] T. Teo, H. Zhu, and S. Chen, et al., "Principle and modeling of a novel moving coil linear-rotary electromagnetic actuator," IEEE Trans. Ind. Electron., vol. 63, no. 11, pp. 6930-6940, Jun. 2016.

[9] T. T. Overboom, J. W. Jansen, and E. A. Lomonova, et al., "Design and optimization of a rotary actuator for a two-degree-of-freedom $z \phi$-module," IEEE Trans. Ind. Appl., vol. 46, no. 6, pp. 2401-2409, Sep. 2010.

[10] O. Dobzhanskyi, E. Amiri and R. Gouws, "Comparison analysis of electric motors with two degrees of mechanical freedom: PM synchronous motor vs induction motor," in Proc. 2016 YSF, Nov. 2016, pp. 14-17.

[11] K. Kluszczynski and M. Szczygiel, "How to convert a factory-manufactured induction motor into rotarylinear motor? part 2 design issues from viewpoint of educational purposes and industrial demands," in Proc. 15th REM, Oct. 2014, pp. 1-6.

[12] J. F. Pan, Yu Zou and N. C. Cheung, "Performance analysis and decoupling control of an integrated rotarylinear machine with coupled magnetic paths," IEEE Trans. Magn., vol. 50, no. 2, Art: 7018804, Feb. 2014.

[13] M. M. Nezamabadi, E. Afjei and H. Torkaman, "Design, dynamic electromagnetic analysis, FEM, and fabrication of a new switched-reluctance motor with hybrid motion," IEEE Trans. Magn., vol. 52, no. 4, Art: 8201708, Apr. 2016. 
[14] Y. Fujimoto, T. Suenaga and M. Koyama, "Control of an interior permanent-magnet screw motor with powersaving axial-gap displacement adjustment," IEEE Trans. Ind. Electron., vol. 61, no. 7, pp. 3610-3619, Jun. 2014.

[15]A. Z. Shukor and Y. Fujimoto, "Direct-drive position control of a spiral motor as a monoarticular actuator," IEEE Trans. Ind. Electron., vol. 61, no. 2, pp. 1063 1071, May 2014.

[16] S. Pakdelian, Y. B. Deshpande and H. A. Toliyat, "Design of an electric machine integrated with transrotary magnetic gear," IEEE Trans. Energ. Conv., vol. 30, no. 3, pp. 1180-1191, Apr. 2015.

[17] Y. Yoshida, M. Mori, W. Kitagawa and T. Takeshita, "Design for cogging torque reduction in two-degree-offreedom cylindrical actuator," in Proc. 2014 Int. Conf. Elect. Mach., Nov. 2014, pp. 478-483.

[18] H. Tsujimoto, S. Tanaka and T. Shimono, et al., "Design and analysis of a resolver for 2DOF tubular motor," in Proc. 42nd ACIES, Oct. 2016, pp. 61696174.

[19] W. Yang and X. Sun, "Magnetic field finite element analysis and thrust characteristics calculation of a linear and rotary stepper motor," in Proc. 2011 ICECE, Oct. 2011, pp. 2311-2314.

[20] B. Assadsangabi and K. Takahata, "Micro rotary-linear actuator assisted by ferrofluid levitation for 3dimensional endoscopic imaging," in Proc. 2015 18th ICSSAM, Jun. 2015, pp. 391-394.

[21]Z. Zhang, H. Zhou and J. Duan, "Design and analysis of a high acceleration rotary-linear voice coil motor," IEEE Trans. Magn., vol. 53, no. 7, Art: 8203509, Jul. 2017.

[22] C. S. Christophe and Y. Fujimoto, "Pre-drive test of an implemented novel radial-gap helical ROTLIN machine," in Proc. 2016 ECCE, Sept. 2016, pp. 1-7.

[23] L. Wang, Y. Wang, and T. Cheng, et al., "Design of a novel rotary-linear pneumatic actuator integrated with ultrasonic motion control mechanism," in Proc. 2015 FPM, Aug. 2015, pp. 417-420.

[24] J. Shin, R. Watanabe, and T. Koseki, et al., "Transverse-flux-type cylindrical linear synchronous motor for large thrust using generic armature cores for rotary machinery," IEEE Trans. Ind. Electron., vol. 61, no. 8, pp. 4346-4355, Jul. 2013.

[25] Y. Zhang, G. Liu, J. Hesselbach. "On development of a rotary-linear actuator using piezoelectric translators," IEEE/ASME Trans. Mech., vol. 11, no. 5, pp. 647-650, Oct. 2006.

[26] T. Mashimo, S. Toyama. "Rotary-linear piezoelectric microactuator with a cubicstator of side length 3.5 mm," IEEE Trans. Ultrason., Ferroel., Freq. Cont., vol. 57, no. 8, pp. 1825-1830, Aug. 2010.

[27] T. Cheng, X. Guo, G. Bao, et. al.. "Analysis and development of plate-attached cylindrical rotary-linear ultrasonic motor," in Proc. 2012 SPAWDA, Nov. 2012, pp. 270-273.

[28] P. Jin, H. Lin, S. Fang and S. L. Ho, "Decoupling control of linear and rotary permanent magnet actuator using two-directional $d-q$ transformation," IEEE Trans. Magn., vol. 48, no. 10, pp. 2585-2591, Oct. 2012.

[29] M. Bertoluzzo, P. Bolognesi, and O. Bruno, et al., "A distributed driving and steering system for electric vehicles using rotary-linear motors," in Proc. 2010 SPEEDAM, Aug. 2010, pp. 1156-1159.

[30] M. Luo, H. Zhou, J. Duan and B. Kou, "Design and analysis of a servo control system for a novel linearrotary voice coil motor," in Proc. 2016 19th ICEMS, Nov. 2016, pp. 1-5.

[31] I. Benţia, M. Ruba and L. Szabó, "On the control of a rotary-linear switched reluctance motor," in Proc. 2011 5th ISCIII, Nov. 2011, pp. 41-46.

[32] L. Szabó, I. Benţia and M. Ruba, "A rotary-linear switched reluctance motor for automotive applications," in Proc. 2012 Int. Conf. Elect. Mach., Nov. 2012, pp. 2615-2621. 